\title{
Extension twin variant selection during uniaxial compression of a magnesium alloy
}

Pei, Y.; Godfrey, A.; Jiang, J.; Zhang, Yubin; Liu, W.; Liu, Q.

Published in:

Materials Science and Engineering: A - Structural Materials: Properties, Microstructure and Processing

Link to article, DOI:

10.1016/j.msea.2012.04.046

Publication date:

2012

Link back to DTU Orbit

Citation (APA):

Pei, Y., Godfrey, A., Jiang, J., Zhang, Y., Liu, W., \& Liu, Q. (2012). Extension twin variant selection during uniaxial compression of a magnesium alloy. Materials Science and Engineering: A - Structural Materials: Properties, Microstructure and Processing, 550, 138-145. https://doi.org/10.1016/j.msea.2012.04.046

\section{General rights}

Copyright and moral rights for the publications made accessible in the public portal are retained by the authors and/or other copyright owners and it is a condition of accessing publications that users recognise and abide by the legal requirements associated with these rights.

- Users may download and print one copy of any publication from the public portal for the purpose of private study or research.

- You may not further distribute the material or use it for any profit-making activity or commercial gain

- You may freely distribute the URL identifying the publication in the public portal 


\section{Elsevier Editorial System(tm) for Materials Science \& Engineering A Manuscript Draft}

Manuscript Number: MSEA-D-11-03530

Title: Extension twin variant selection during uniaxial compression of a magnesium alloy

Article Type: Research Paper

Keywords: Magnesium alloys; twinning; variant selection; EBSD; crystal plasticity

Corresponding Author: Prof. A. Godfrey,

Corresponding Author's Institution: Tsinghua University

First Author: Ying Pei

Order of Authors: Ying Pei; A. Godfrey; Jia Jiang; Yubin Zhang; Wei Liu; Qing Liu

Abstract: Samples of the magnesium alloy AZ31 have been deformed by compression to strains of 5\% and $10 \%$ and microstructural observations made to investigate the activation of specific $\{10-12\}$ extension twin variants. The twinning has been analyzed on a grain-by-grain basis for more than 260 grains to determine both the number of extension twin variants in each grain, and the volume fraction of each. At $5 \%$ strain approx. $30 \%$ of the grains contain twins corresponding to variants with the third or lower ranked Schmid factor, with the fraction increasing to $40 \%$ after $10 \%$ compression. A grain size effect is also observed in that smaller grains are less likely to contain lower ranked twin variants. For both $5 \%$ and $10 \%$ compression no clear relationship exists between the volume fraction of each twin variant in a given grain population and the Schmid factor for the twin variant. A positive linear relationship can be defined, however, between the maximum twinning fraction that a twin variant can reach and its Schmid factor. 
TITLE PAGE

Extension twin variant selection during uniaxial compression of a magnesium alloy

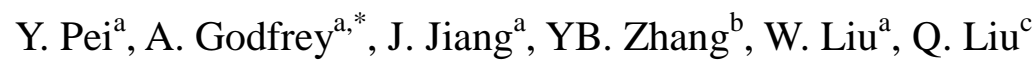

a. Laboratory for Advanced Materials, Dept. Materials Science and Engineering, Tsinghua University, Beijing 100084, China.

b. Danish-Chinese Center for Nanometals, Materials Research Division, Ris $\varnothing$ National Laboratory for Sustainable Energy, Technical University of Denmark, DK-4000, Roskilde

c. College of Materials Science and Engineering, Chongqing University, Chongqing 400045, China.

*Corresponding author:

Laboratory for Advanced Materials, Dept. Materials Science and Engineering, Tsinghua University, Beijing 100084, China.

awgodfrey@mail.tsinghua.edu.cn

Tel: +861062788317; FAX: +861062771160 


\title{
Extension twin variant selection during uniaxial compression of a magnesium alloy
}

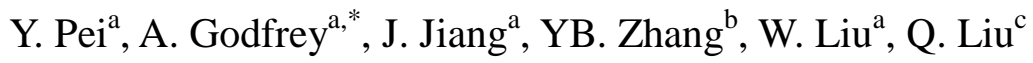

a. Laboratory for Advanced Materials, Dept. Materials Science and Engineering, Tsinghua University, Beijing 100084, China.

b. Danish-Chinese Center for Nanometals, Materials Research Division, Ris $\varnothing$ National Laboratory for Sustainable Energy, Technical University of Denmark, DK-4000, Roskilde

c. College of Materials Science and Engineering, Chongqing University, Chongqing 400045, China.

*Corresponding author:

\begin{abstract}
:
Samples of the magnesium alloy AZ31 have been deformed by compression to strains of 5\% and $10 \%$ and microstructural observations made to investigate the activation of specific $\{101$ 2 ) extension twin variants. The twinning has been analyzed on a grain-by-grain basis for more than 260 grains to determine both the number of extension twin variants in each grain, and the volume fraction of each. At 5\% strain approx. $30 \%$ of the grains contain twins corresponding to variants with the third or lower ranked Schmid factor, with the fraction increasing to $40 \%$ after $10 \%$ compression. A grain size effect is also observed in that smaller grains are less likely to contain lower ranked twin variants. For both $5 \%$ and $10 \%$ compression no clear relationship exists between the volume fraction of each twin variant in a given grain population and the Schmid factor for the twin variant. A positive linear relationship can be defined, however, between the maximum twinning fraction that a twin variant can reach and its Schmid factor.
\end{abstract}

Keywords: Magnesium alloys, twinning, variant selection, EBSD, crystal plasticity. 


\section{Introduction}

Twinning in magnesium alloys plays an important role in plastic deformation during low and medium temperature mechanical forming operations [1-6] by providing additional independent deformation modes. The operation of twinning during deformation also has a strong effect on the flow stress and on texture evolution, as a result of the lattice re-orientation that accompanies twinning [7]. Among all the possible twinning modes in $\mathrm{Mg}$ alloys $\{1012\}$ "extension" twinning (so-called as it results in an elongation of the unit cell along the $c$-axis direction) is the most commonly observed, on account of the low critical resolved shear stresses (CRSS) [8] for this twinning mode.

The grain orientation dependence of twinning within individual grains has been discussed by several authors in terms of a Schmid factor (SF) [1,9-16] analogous to that used in the analysis of dislocation glide. Although in many cases primary twinning has been found to show an general correspondence with the Schmid factor, significant deviations from this have been reported, both for $\{1012\}$ primary twinning in high-purity magnesium and zirconium [15,16], and more recently for $\{1011\}$ compression twinning in a magnesium alloy [13]. Additionally a number of studies have shown that "non-Schmid" behavior is a characteristic feature of $\{1012\}$ secondary twinning, where the twinning variant selection has been explained in terms of a requirement for local accommodation between the primary $\{1011\}$ twin and the secondary $\{1012\}$ twin $[1,14]$.

One interesting aspect of $\{1012\}$ extension twinning that has received less attention is the observation that in many grains multiple twin variants are observed. This is important as such multiple twinning influences both the texture evolution during deformation, and microstructural subdivision due to the intersection of different twin variants. In particular 
questions still remain open regarding the ability of a SF criterion to predict the type, combination, and fraction of twinning variants in individual grains where multiple variants are seen. In this paper we present therefore an analysis of primary $\{1012\}$ extension twinning based on a grain-by-grain investigation of the twinning in a large number of grains, using data from electron backscatter diffraction (EBSD) investigations of a AZ31 Mg-alloy deformed by uniaxial compression. Other grain-by-grain studies have looked at the number and thickness of twins, independent of variant type, with a focus on the twin nucleation and growth mechanisms $[15,16]$. Here the main parameter we focus on is the twin volume fraction, as this represents the microstructural contribution to the applied strain for each twin variant and can be directly used therefore in guiding the development of improved crystal plasticity models.

\section{Experimental Methods}

The material employed was a commercially available AZ31 (Mg-3Al-1Zn-0.3Mn in wt.\%) sheet containing nearly equiaxed grains of an average linear intercept size $\sim 14 \mu \mathrm{m}$ and a strong $\{0001\}$ basal plane texture (Fig.1). Cylinders of size $8 \mathrm{~mm}$ diameter and $12 \mathrm{~mm}$ height were cut from the sheet for the uniaxial compression testing with the compressive axis parallel to transverse direction (TD) of the rolled sheet, so that the $<0001>$ directions of most grains were oriented nearly perpendicular to the compressive axis, and accordingly $\{1012\}$ twinning could be activated easily during compression.

Compression testing was carried out at $150{ }^{\circ} \mathrm{C}$ at a constant strain rate of $0.01 \mathrm{~s}^{-1}$ using a Gleeble $1500 \mathrm{D}$ in a vacuum of $1 \times 10^{-3} \mathrm{~Pa}$. After deformation, the samples were cut in the mid-plane, and then the exposed sections were ground to 4000 grit $\mathrm{SiC}$ paper before final electro-polishing (Struers ACII electrolyte; $20 \mathrm{~V}$ and $20^{\circ} \mathrm{C}$ for 1 minute). Microstructural data 
were collected using EBSD, which was performed in beam-control mode using a TESCAN 5136XM scanning electron microscope equipped with an Oxford Instruments-HKL Channel 5 EBSD system. Representative regions were mapped using a step size of $1 \mu \mathrm{m}$ over areas of $400 \mu \mathrm{m} \times 400 \mu \mathrm{m}$.

\section{Twin variant analysis}

\subsection{Twin variant identification}

The twinning mode can be directly determined from the EBSD data as a result of the unique crystal misorientation associated with each twinning mode (for example, a misorientation of $86^{\circ}$ about a $<1120>$ axis results from $\{1012\}$ extension twinning). In this investigation a key objective is to determine the particular twinning variant among the six equivalent $\{1012\}$ twinning systems. It is worth adding here that labeling a twin as a particular variant is by itself an arbitrary operation (due to the action of crystal symmetry). However, once the parent orientation description is fixed, then different variants can be labeled in a meaningful way, and more importantly these variants can be compared with other properties (e.g. in this study the Schmid factor for twinning) based also on the fixed parent orientation.

The identification of twinning $\{1012\}$ variants was carried out based on the methods described in [17]. For convenience these are summarized briefly here. For cases where the twin boundary plane was clearly identifiable a trace analysis was used to identify the twin variant. For grains where the extent of twinning was such that the trace method could not be used, the twin variant was identified by comparing the experimental twin variant orientation with the six possible twin orientations calculated based on the parent grain orientation and then finding the closet match. Previous work [17] has shown that this method is sufficiently accurate to determine the exact twin variant in samples deformed up to $10 \%$ in compression 
if the deviation between the observed twin orientation and the calculated twin orientation is less than $3^{\circ}$. For a few cases where the smallest deviation was greater than this value these twins were not included in the subsequent data analysis. For grains where more than one twin variant was seen and where no parent material remained a parent-daughter grain orientation reconstruction method was used to determine the initial grain orientation and hence the twin variants [17].

\subsection{Schimd factor}

The SFs corresponding to the twinning variants were calculated on the assumption that the stress state of each grain coincided with the macroscopic applied stress. Based on these calculations the SF rank (1 highest; 6 lowest) of each observed twinning variant was identified. The sign of the SF was defined here such that a positive value corresponds to twinning giving an elongation along the $c$-axis.

\subsection{Twin variant pairs}

Although the methods described above can be used to identify the specific twin variant we also refer in the present paper to twin variant pairs, corresponding to twinning on "opposite" twinning planes, as shown in Fig. 2a. The six possible extension twin variants from any parent orientation can be grouped into three such pairs. The relationship between the parent orientation and the six possible twinning variants are shown in Fig. 2b, which illustrates that the two twin variants in the same pair have a characteristic misorientation of $7.4^{\circ}$ (correspondingly with similar SF values), while two variants from any different pairs have a misorientation of either $60.0^{\circ}$ or $60.4^{\circ}$.

\subsection{Twin volume fraction}

In this work the main parameter used to quantify twinning during compression is the twin 
volume fraction. In other recent studies $[15,16]$ the twin number and thickness have been used as quantitative parameters for the characterization of twin nucleation and growth. One complication, however, with these parameters with regard to the present study is that even after strains of a few $\%$ the number of twins per grain is observed to decrease, as a result of lateral growth and abutment of twins of the same variant. The twin volume fraction is therefore a more direct measure of the microstructural response to loading as this parameter can be related directly, via the Schmid factor, to the contribution to the external strain from the activated twin variants.

Although it is possible to determine the true twin shape within each grain using either serial sectioning [18] or a 3D-XRD approach [19], both of these methods are time consuming and require complicated apparatus. In order therefore to estimate the twin extent (variant volume fraction) within each grain some assumptions were made concerning the 3-dimensional geometry of the twin to allow the use of standard stereological methods [20]. For twins with a well defined twin plane the volume fraction was determined by considering the twin as a sheet of material inclined to the viewing surface ( $\{1012\}$ twins in $\mathrm{Mg}$ are only slightly lens shaped and so this is a good approximation). The twin volume fraction was therefore calculated as $A_{T} \sin \theta$, where $A_{T}$ is the twin area fraction in the viewing plane and $\theta$ is the angle between the twin plane and the viewing plane. For grains where the twinning was more extensive (typically more than $80 \%$ twinned area fraction) so that twin plane was no longer well defined it was assumed that the volume fraction was equal to the observed area fraction.

\section{Results}

\subsection{Microstructure and texture}


A sigmoidal hardening was observed in the flow stress curve during compression (Fig. 3). The shape of the flow curve suggests twinning as the dominant deformation mode in the early stages of plastic deformation. For this investigation, where the focus is on $\{1012\}$ twinning, samples were taken for microstructural examination at strains of $5 \%$ and $10 \%$. Typical microstructures at both strains, together with the associated pole figures, are shown in Fig. 4.

In the $\varepsilon=5 \%$ sample it is seen that $\{1012\}$ twinning occurs in most grains, and that in some grains several $\{1012\}$ twin variants can be found (in the EBSD maps $\{1012\}$ twin boundaries are shown in red). The peak near the compression axis (CA) in the $\{0001\}$ pole figure corresponds to the orientations resulting from these twins. In the $10 \%$ reduction sample fewer $\{1012\}$ twin boundaries are seen (Fig. 4b). This is because twin widening takes place with increasing compression and results in an increase in the twinned area fraction but a reduction in the total number (and boundary length per unit area) of the twins. In accordance with this in the $\{0001\}$ pole figure the peak near CA is considerably enhanced. In both EBSD maps many boundaries indicating the abutment of two $\{1012\}$ variants from different pairs (shown in blue in Fig.4) are seen, while the abutment of two twin variants from the same pair (shown in purple) is less common.

\subsection{SF rank}

From the EBSD data 136 grains, containing 248 \{1012\} twinning variants, were selected at random from the 5\% deformed sample, and 132 grains, containing $216\{1012\}$ twinning variants, were selected from the $10 \%$ deformed sample for a detailed Schmid factor analysis of the twinning behavior. In the present work, instead of focusing on the distribution of SF values $[15,16]$, we concentrate instead on an analysis of the number and types of variants in 
individual grains, with the objective of developing a more thorough description of strain accommodation via twinning in order to improve crystal plasticity models for deformation of Mg-alloys.

The SF rankings of the observed twinning variants in these individual grains are summarized in Fig.5. After 5\% deformation (Fig. 5a) approx. one third of the grains only contained one twinning variant, and in most cases this corresponded to the variant with the first or second highest SF. In many grains however more than one variant was seen and these additional variants did not show a clear relationship with the SF ranking.

The results of the $10 \%$ strained sample in Fig.5(b) were similar, although with one striking difference, namely that the fraction of grains containing only two twins, with the first and second highest SF, was observed to decrease (from approx. $33 \%$ at $5 \%$ reduction to $11 \%$ after $10 \%$ reduction). One factor for this decrease could be the operation of additional twin variants (so that the grains are counted in a different bin in Fig. 5). However this cannot account for the large observed difference. In fact the decrease is related to the difficulty in identifying both twin types of a twin pair at a strain of $10 \%$, particularly when a large fraction of the grain has twinned.

This problem is illustrated in Fig. 6. At 5\% reduction two twin variants, corresponding to twins from the same pair can be clearly identified by the misorientation between the twins $\left(7.4^{\circ}<1210>\right)$ and by the twin trace angles (Fig. 6a). At $10 \%$ strain however a more common observation is that grains are almost fully twinned and only in some cases can small segments of boundary corresponding to $7.4^{\circ}<1210>$ misorientation be identified, as shown in Fig. $6 \mathrm{~b}$ (defined here with a tolerance of $3^{\circ}$ on the angle and $15^{\circ}$ on the rotation axis). Closer inspection however of a band contrast image for such grains can show "ghost" traces 
of the two different twin types, where these traces match the expected values for the two twins with highest Schmid factors for this grain.

This observation suggests that in addition to continued twin development after impingement of the twins [21] the twinned material rotates in such a way to reduce the misorientation angle between the two twins. Further work to explore this process is underway. The observed decrease in the number of two-twin same-pair grains is therefore artificial and highlights the need to consider the twins in pairs at a reduction of $10 \%$ even when a specific variant can be identified from the observed misorientation or twin trace angle.

\subsection{Twinned volume fraction vs. Schmid factor}

A total of 49 grains containing 76 twinning variants in the 5\% strained sample and 38 grains containing 54 variants in the $10 \%$ strained sample were picked out randomly and the twinning volume fraction versus SF for each twinning variant plotted. The results are shown in Fig. 7. In Fig. 7 black lines are drawn to connect the variants in grains containing two twinning variants, and orange lines are drawn to connect values for grains containing three variants.

Instead of a simple positive linear relationship between the twinning volume fraction and SF, a complex distribution in seen in the 5\% strained sample. Although the average thickness of twins at this strain level has been reported to increase with increasing SF in high-purity $\mathrm{Mg}$ [15], the data in Fig. 7 show that a large spread in volume fraction exists for twins with similar SF values. In particular for grains containing only a single twin variant a wide range of SFs is seen, and for grains with large SFs in the range 0.4 0.5 the twinned volume fractions range from almost $0 \%$ to $80 \%$. A similar, though still more complex pattern is seen for the data collected at $10 \%$ strain (Fig. $7 b$ ). However, at this strain most single twin variant 
grains with SF values of between 0.3 and 0.5 were observed to be nearly fully twinned.

For grains containing more than one variant it can be expected that the black and orange lines should have positive slope (corresponding to a higher volume fraction for the twin variant with a higher SF value). This is seen, but there are some exceptions to this pattern. At 5\% strain for differences between the two SFs corresponding to two twin variants in one grain of larger than 0.04 (18 grains in total), the slopes of 16 lines were positive, indicating that twinning variants with higher SF progressed to a larger extent. For cases where the difference was smaller than 0.04 , the slope was either negative (6 lines) or positive (8 lines). The situation in the $10 \%$ strained sample was similar. One possible explanation is that when SFs of more than one variant are similar the local stress state plays a controlling role in determining which of the two variants grows preferentially.

Nevertheless, despite the lack of a direct relationship between twinned volume fraction and Schmid factor, an approximate upper limit representing the maximum twinned fraction as a function of SF at each strain can be drawn (shown in the figures by the line drawn in blue), i.e. the volume fraction of twins with a given SF value can vary between 0 and a fixed maximum value. The upper limit line is shifted to the left with increasing strain, reflecting an increase in the number of twin variants with low SFs at larger strains.

\subsection{Untwinned grains}

At 5\% strain some grains were observed in which no twin boundaries were seen. Some of these grains were in fact fully twinned - however such grains were readily identified from their orientation and were excluded from the following analysis. The untwinned grains were analyzed in terms of the maximum SF value for twinning. The results are plotted in Fig. 8 as histograms showing the distribution of the maximum SF values for the untwinned grains. It 
can be seen that even for grains with a maximum SF of $0.3 \sim 0.5$, a total of 10 untwinned grains out of $\sim 300$ grains were seen in the 5\% strained sample. All the untwinned grains have areas smaller than $300 \mu \mathrm{m}^{2}$ with most grains having an area of less than $100 \mu \mathrm{m}^{2}$. After a compression of $10 \%$ only a very small number of grains with SF $0.3 \sim 0.5$ were left without evidence of twinning. Yang et al. [22] also reported a similar result by the tracking of a single grain with increasing strain, whereas the present work confirms this pattern based on observation of a large number of individual grains.

\section{Discussion}

\subsection{Variant selection mechanisms}

The results presented in Fig. 5 for a large number of grains show that the number and combination of twin variants in each individual grain can be quite complicated. Twin variants with the first and/or second highest SF can operate in nearly all grains, and variants with third and/or fourth ranked SF appear in almost 1/3 of the grains (Fig. 5). A few grains also contain variants with the fifth and/or sixth ranked SF (4 out of 132 grains in the $10 \%$ strained sample). This is in contrast to the observation of Hong et al [9], where they reported that during compression perpendicular to the c-axis mostly only one twin variant or twin variant pair was active in each grain.

To examine further the factors controlling twin variant selection, and in particular the operation of twin variants with the third and/or fourth ranked SF, grains in the $10 \%$ strained samples were divided into two groups (excluding here the 4 grains containing twin variants with fifth and/or sixth ranked SF) according to (a) grains containing variants with third and/or fourth rank SF, and (b) grains containing only variants with first and/or second rank SF. The untwinned orientations of grains in each group are plotted in Fig. 9. The figure 
shows that grains containing twin variants with third and/or fourth rank SF trend to locate near the [1210] corner of the inverse pole figure. A less well defined distribution is seen for grains with only the first and/or second ranked SF twin variants, though some clustering towards the <1010> axis still can be discerned. It is notable however there is no clear boundary separating the compression axis directions for these two groups of grains.

\subsubsection{Grain orientation}

In order to examine in more detail the effect of grain orientation on twin variant operation, contour plots showing the variation in SF value as a function of compression axis direction have been calculated. Considering each grain has three pairs of possible variants, and that the two variants in each pair have similar SFs, plots are only shown for the first, fourth and sixth highest SF variants (Fig. 10(a-c)). An inverse pole figure showing the compression axis directions of the material investigated is shown in Fig. 10d. It can be seen that the highest SFs of most grains range from 0.3 to 0.5 , whereas the sixth ranked SFs are all near zero, and therefore not expected to operate. However, the fourth ranked SFs are spread over a wide range of $0.1 \sim 0.4$.

To further analyze cases where twinning occurs on variants with the third and/or fourth highest SF, we define the parameter $\alpha$ as the ratio of the fourth highest SF to the highest SF for each grain orientation. When $\alpha$ is large, four of the six SFs are similar (for compression along the exact [1210] direction, $\alpha=1$ and the Schmid factors are equal on four twin variants), whereas a small value of $\alpha$ means twinning is only expected on one, or both, of the pair with the highest SFs. Contours showing the variation of $\alpha$ with compression axis direction are marked in Fig. 9, which also shows the compression direction of grains observed experimentally to contain (Fig. 9a) or not contain (Fig. 9b) twin variants with the 
third and/or fourth ranked SF. It is seen that the parameter $\alpha$ provides a guide to operation of lower ranked twin variants: grains where third and/or fourth ranked variants are observed have large values of $\alpha$, with almost all such grains having the $\alpha$ value above 0.4 and many grains above 0.6. However, this relationship is only approximate, and as shown in Fig. 7 the extent of twinning varies greatly as a function of Schmid factor on a grain by grain basis. Other factors that may influence the twinning are therefore considered in the following sections.

\subsubsection{Grain size effect on multiple variant twinning}

It has already been reported in many studies of AZ31 that there is a grain size effect on twinning, with a threshold grain size below which twinning does not take place (typically reported to be in the range from $4-8 \mu \mathrm{m}$ at $150^{\circ} \mathrm{C}$ [23]). In Section 4.4 it has already been noted that twin nucleation is delayed in grains with areas in the viewing section of up to $100 \mu \mathrm{m}^{2}$ (corresponding to an equivalent circle diameter of approx. $10 \mu \mathrm{m}$ ).

The results of the present study also show that the occurrence of multiple variant twinning is also partly dependent on the grain size. As described above, for a compression of $10 \%$ grains where the third and/or fourth ranked SF variants are seen as a second twin variant predominantly have $\alpha$ values larger than 0.6. However, the converse is not true $-\mathrm{a}$ significant number of grains with $\alpha$ above 0.6 do not contain such third and/or fourth ranked variants (Fig.10b). Figure 11 shows the distribution of grain areas for all grains with compression axis directions giving values of $\alpha$ above 0.6 , showing separately grains where lower ranked SF variants are either seen, or not seen. It is immediately clear that almost all of the grains with $\alpha$ above 0.6 that do not show lower ranked twin variants have the smallest grain sizes. Data have also been analyzed for the $5 \%$ deformed sample, where a similar 
result is obtained. It can be concluded that in smaller grains fewer variants are observed compared to grains of similar orientation but larger size, and thus the grain size dependence on twinning also extends to the operation of lower ranked variants. This conclusion is in line with the observations of Barnett [24], Capolungo et al. [16] and Beyerlein et al. [15], who suggested that the number of twins per grain noticeably increases with grain area, although in these publications the specific twin variant types were not reported.

\subsection{Dependence on the local stress conditions}

The analysis of $\{1012\}$ twin variants shows that the SF value provides an approximate guide for which particular twin variants will operate. However, the results also show that a significant variation exists between grains of similar orientation and size, and that the extent of twinning is not related in a simple matter to the SF value for a given twin variant. One possible reason for this is a variation of the local stress state, due both to a variation in the plastic strain supported by each grain, and to internal stresses resulting from nearby twins, or from slip processes within the untwinned parts of a grain [13]. Related arguments, based on self-strain accommodation have been proposed to account for the variant selection during double twinning in Mg-alloys [1,14,25]. Based on in-situ 3D-XRD observations Aydiner et al. have reported that the stress state within a twin can be significantly different from that in the untwinned matrix [19]. For the grain tracked by Aydiner et al. the stresses within each twin variant were observed to increase with increasing strain, though the stress values within each variant were different and differed to the applied macroscopic stress. The stress of one variant was especially low in the low strain stage. Their results are therefore accordant with the complex pattern of seen in Fig.7(a).

It is perhaps worth noting that some in-situ neutron diffraction results show that twinning 
proceeds at a rate that can be correlated with the SF value (see Fig. 5 and 6 in Ref. [26]). This observation does not conflict however with the present work, as the neutron diffraction data represent the summed behavior for a large population of twins with the same SF, and not, as in the current study, the behavior of individual grains.

The results presented in this study, and particularly those shown in Fig. 7, suggest that the detailed twinning behavior can only be described in a statistical manner and that it is difficult to predict the extent of any particular twin variant only based on the SF value for that variant based on the macroscopically applied stress state. Nevertheless the results provide support for an upper limit on the correlation between the twinning population (number or variants and extent of each variant) and the SF value. Twinning rules based on these are expected to be useful for the development of improved polycrystal plasticity models aimed at the prediction of both the microstructure and macroscopic texture evolution during plastic deformation of magnesium alloys.

\section{Conclusions}

The operation of extension twinning during uniaxial compression of AZ31 has been investigated in samples aligned such that twinning is the preferred deformation mode. The main observations and conclusions are summarized below:

(1) After uniaxial compression to a strain of 5\% approx. $30 \%$ of the grains contain extension twins corresponding to systems with the $3^{\text {rd }}$ or lower Schmid factor ranking. This figure increases to $40 \%$ for samples deformed to $10 \%$ compression.

(2) Grain size can influence the twinning in a number of ways. At low strains twinning is delayed in the smallest grains. At higher strains twinning is seen in most grains, but for a given grain compression direction fewer variants are seen in grains of smaller size but 
similar orientation.

(3) For the strain levels examined in this study (5\% and $10 \%$ compression) no fixed relationship exists between the volume fraction of each twin variant in a given grain population and the Schmid factor for the twin variant. A positive linear relationship can be defined, however, between the maximum twinning fraction that a twin variant can reach and its Schmid factor.

\section{Acknowledgements}

The authors acknowledge support for this work from the National Key Basic Research Program of China (Grant No. 2007CB613703) and from the Danish National Research Foundation and the National Natural Science Foundation of China (Grant No. 50911130230) for the Danish-Chinese Center for Nanometals, within which part of this work was performed.

\section{References}

[1] M.R. Barnett, Z. Keshavarz, A.G. Beer, X. Ma, Acta Mater. 56 (2008) 5-15.

[2] L. Jiang, J.J. Jonas, R.K. Mishra, A.A. Luo, A.K. Sachdev, S. Godet, Acta Mater. 55 (2007) 3899-3910.

[3] M.R. Barnett, Z. Keshavarz, M.D. Nave, Metall Mater Trans A. 36A (2005) 1697-1704.

[4] M.R. Barnett, Mater Sci Eng A. 464 (2007) 1-7.

[5] M.R. Barnett, Mater Sci Eng A. 464 (2007) 8-16.

[6] S.W. Xu, K. Oh-ishi, S. Kamado, T. Homma, Scripta Mater 65 (2011) 875-878.

[7] J.W. Christian, S. Mahajan, Prog Mater Sci. 39 (1995) 1-157.

[8] R.E. Reedhill, W.D. Robertson, Acta Metall. 5 (1957) 717-727.

[9] S.G. Hong, S.H. Park, C.S. Lee, Acta Mater. 58 (2010) 5873-5885.

[10] S. Godet, L. Jiang, A.A. Luo, J.J. Jonas, Scripta Mater. 55 (2006) 1055-1058.

[11] C.S. Hong, N.R. Tao, K. Lu, X. Huang, Scripta Mater. 61 (2009) 289-292. 
[12] J.F. Bingert, T.A. Mason, G.C. Kaschner, P.J. Maudlin, G.T. Gray, Metall Mater Trans A. 33 (2002) 955-963.

[13] J.J. Jonas, M. S., T. Al-Samman, G. Gottstein, L. Jiang, E. Martin, Acta Mater. 59 (2011) 2046-2056.

[14] E. Martin, L. Capolungo, L. Jiang, J.J. Jonas, Acta Mater. 58 (2010) 3970-3983.

[15] I.J. Beyerlein, L. Capolungo, P.E. Marshall, R.J. McCabe, C.N. Tome, Philos Mag. 90 (2010) 2161-2190.

[16] L. Capolungo, P.E. Marshall, R.J. McCabe, I.J. Beyerlein, C.N. Tome, Acta Mater. 57 (2009) 6047-6056.

[17] J. Jiang, A. Godfrey, W. Liu, Q. Liu, Scripta Mater. 58 (2008) 122-125.

[18] A.J. Schwartz, M. Kumar, B.L. Adams, Electron Backscatter Diffraction in Materials Science, Kluwer Academic/Plenum Publishers, New York, 2000.

[19] C.C. Aydiner, J.V. Bernier, B. Clausen, U. Lienert, C.N. Tome, D.W. Brown, Phys Rev B. 80 (2009) 024113.

[20] T.A. Mason, J.F. Bingert, G.C. Kaschner, S.I. Wright, R.J. Larsen, Metall Mater Trans A. 33 (2002) 949-954.

[21] M.D. Nave, M.R. Barnett, Scripta Mater. 51 (2004) 881-885.

[22] H.J. Yang, S.M. Yin, C.X. Huang, Z.F. Zhang, S.D. Wu, S.X. Li, Y.D. Liu, Adv Eng Mater. 10 (2008) 955-960.

[23] M.R. Barnett, Z. Keshavarz, A.G. Beer, D. Atwell, Acta Mater. 52 (2004) 5093-5103.

[24] M.R. Barnett, Scripta Mater. 59 (2008) 696-698.

[25] D. Ando D, J. Koike, Y. Sutou, Acta Mater. 58 (2010) 4316-4324.

[26] O. Muransky, M.R. Barnett, D.G. Carr, S.C. Vogel, E.C. Oliver, Acta Mater. 58 (2010) 1503-1517. 


\section{Figure Captions:}

Figure 1: $\{0001\}$ and $\{1010\}$ pole figure showing the initial texture of the sample investigated

Figure 2: (a) Schematic of the magnesium unit cell showing two "opposite" $\{1012\}$ twinning planes ( $\mathrm{C} 1$ and $\mathrm{C} 2$ in red) forming a twinning pair. Only the traces in the $\{0001\}$ plane for the other four twinning planes are shown for clarity; (b) The orientation of one parent matrix (black triangle) and corresponding six $\{1012\}$ twinning variants (squares) in a $\{0001\}$ pole figure. Twin variants with the same colors (but different brightness) show twins from the same pair with a characteristic misorientation of $7.4^{\circ}$, while the two variants from any different pairs have also misorientations of either $60.0^{\circ}$ or $60.4^{\circ}$

Figure 3: Flow curves during uniaxial compression testing of the samples at $150^{\circ} \mathrm{C}$

Figure 4: Microstructure and $\{0001\}$ pole figure (compression axis at the centre) for samples after uniaxial compression at $150{ }^{\circ} \mathrm{C}$ to strains of: (a) $5 \%$;(b)10\%. Red lines indicate $\{1012\}$ twins with characteristic misorientation $86.4^{\circ}$; blue lines indicate boundaries between two twin variants from different pairs; purple lines indicate boundaries between two variants from the same twin pair; thin black lines indicate other boundaries with misorientations from $2^{\circ}$ to $10^{\circ}$; thick black lines indicate above misorientations $>10^{\circ}$. Solid black areas indicate non-indexed regions in the EBSD map.

Figure 5: Ranking of Schmid factors for twin variants in samples deformed to strain of (a) $5 \%$, (b) $10 \%$. Example of labelling: Rank "1" represents a grain with a single twin variant with the highest SF; Rank "14" denotes a grain with two twin variants corresponding to the first and fourth highest Schmid factors

Figure 6: (a) Example EBSD boundary map of a grain at strain of 5\% containing two twin 
variants from the same pair; (b) example EBSD boundary map of almost fully twinned grain at a strain of $10 \%$. In a few places $7.4^{\circ}<1 \overline{2} 10>$ boundaries are seen indicating abutment of two twins from the same pair. For both maps "M" indicates the untwinned matrix; (c) EBSD band contrast map for same grain as (b) showing "ghost traces" corresponding to the two twin variants. Color code for misorientation same as Fig. 4.

Figure 7: Relationship between twinning volume fraction and Schmid factor at strains of: (a) $5 \%$; (b) $10 \%$. Black lines connect the variants in grains containing two twinning variants; orange lines connect twins in grains containing three variants. An envelope giving the maximum twinning volume fraction as a function of Schimd factor is plotted in aqua.

Figure 8: The maximum Schmid factor for untwined grains after compresion at $150{ }^{\circ} \mathrm{C}$ to strains of: (a) 5\%; (b) $10 \%$.

Figure 9: Distribution of grain orientations after 10\% deformation for :(a) grains containing twinning variants with third and/or fourth highest Schmid factor (SF); (b) grains only containing twinning variants with first and/or second highest SF. Contours of the ratio of fourth ranked SF to first ranked SF are also shown.

Figure10: Inverse pole figures (compression axis direction) showing contour plots of the variation in Schmid factor (SF) value for the: (a) highest SF, (b) fourth highest SF, (c) sixth highest SF; (d) the orientations of grains for a sample of the initial material used in this study.

Figure 11: Distribution of grain area for all the grains with compression axis directions corresponding to an $\alpha$ value $>0.6$, where $\alpha$ is the ratio of the fourth ranked SF to the first ranked SF. 


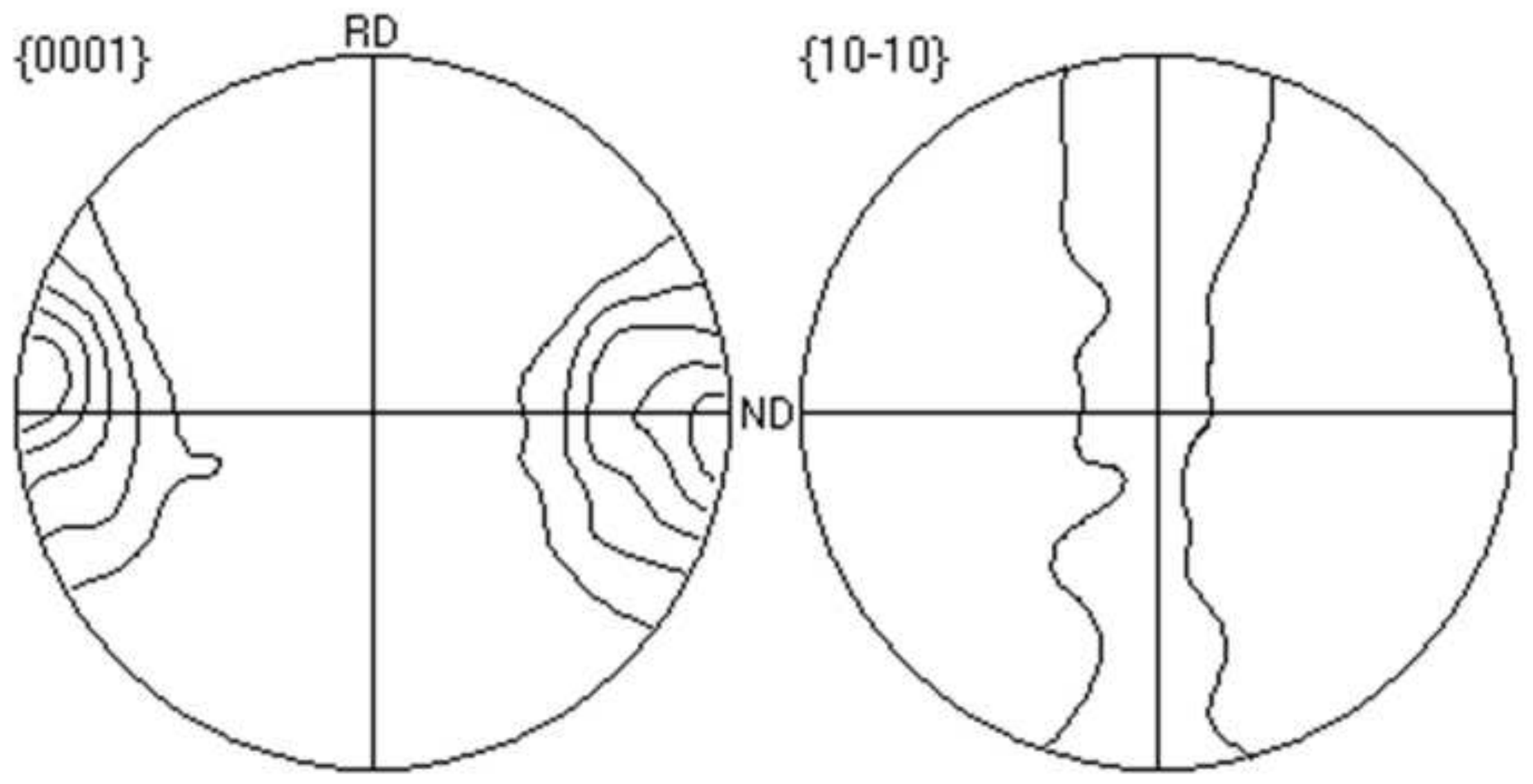

Figure 1: $\{0001\}$ and $\{10-10\}$ pole figures showing the initial texture of the sample investigated 
a)

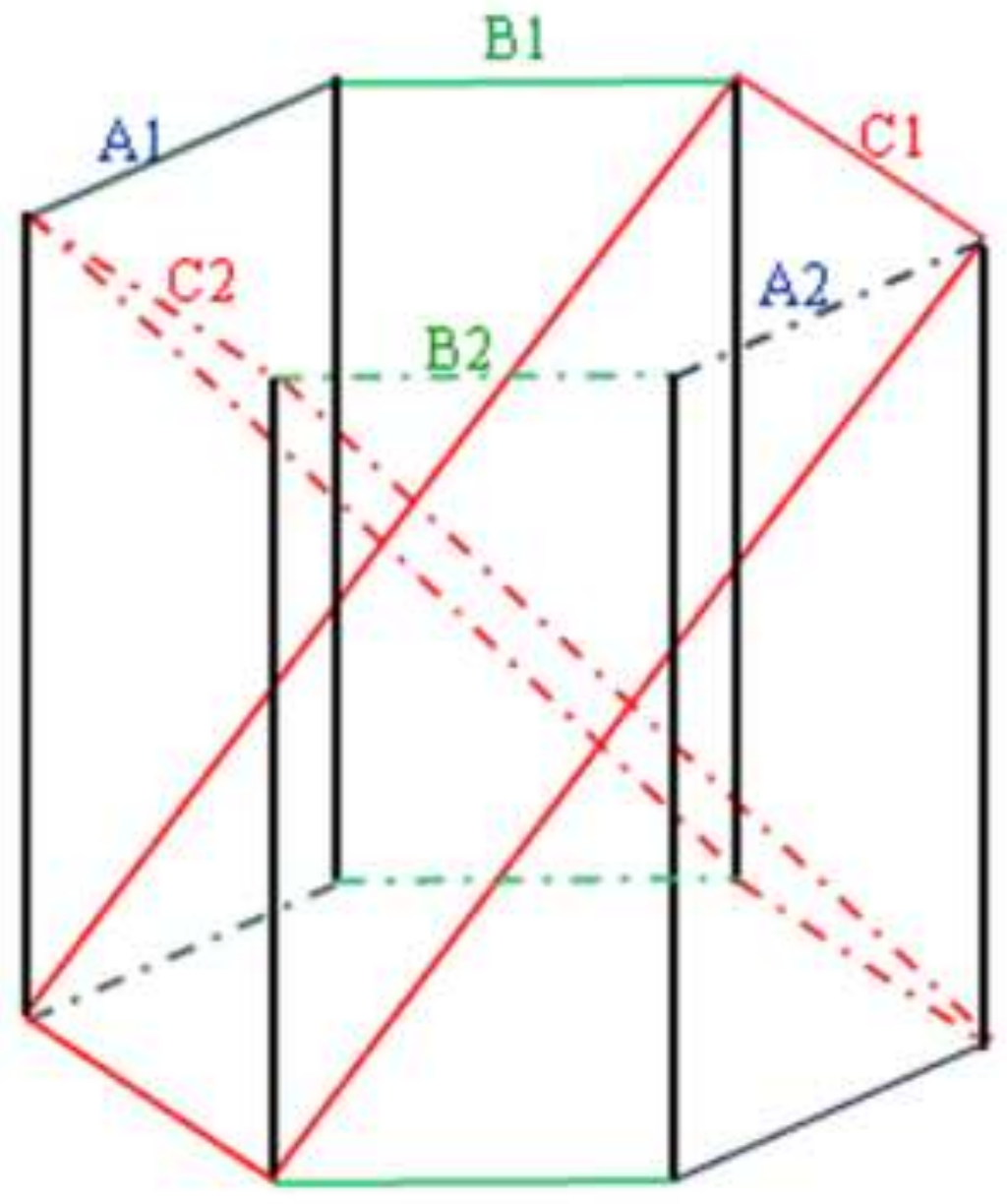

b)

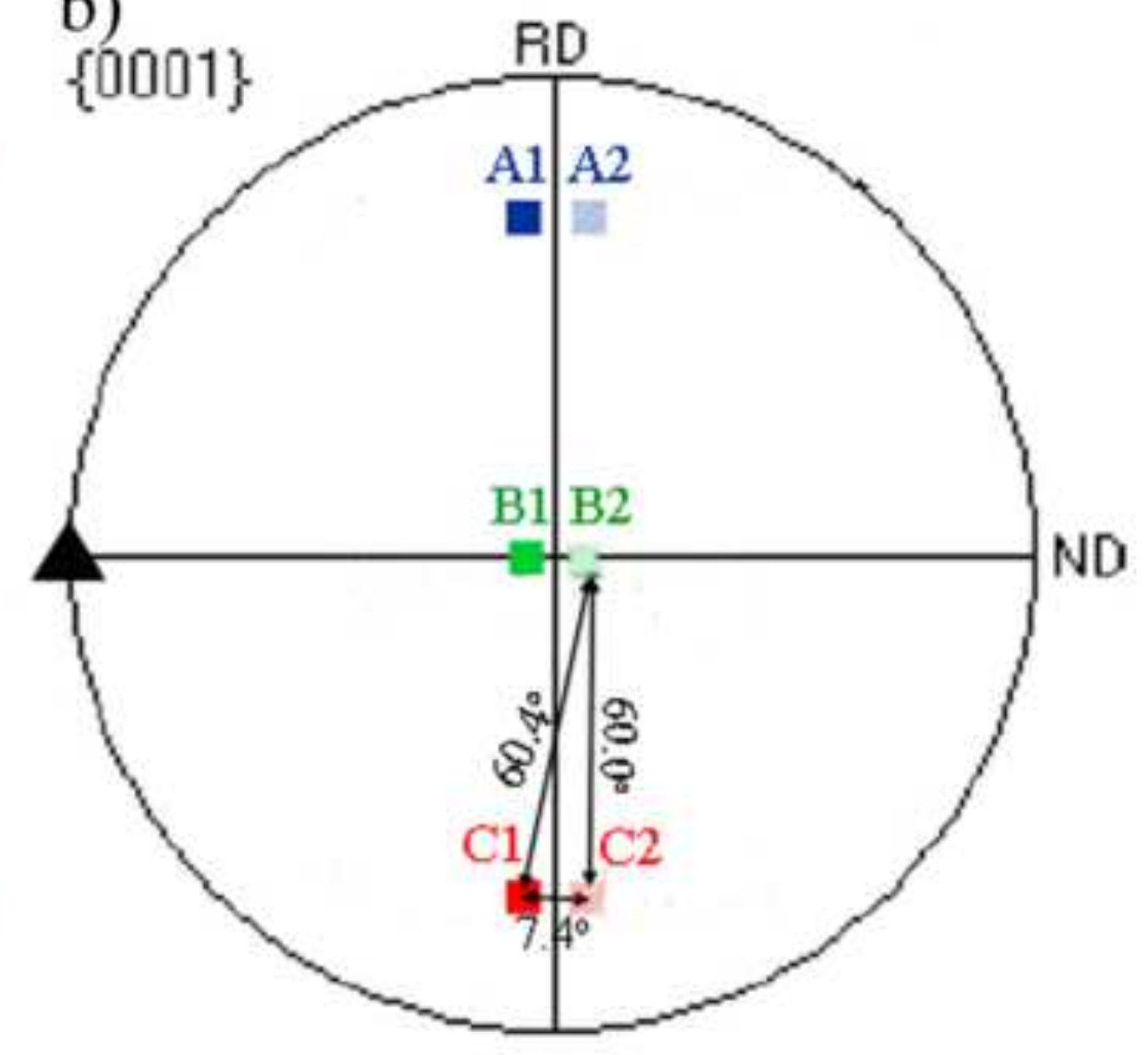

Figure 2: (a) Schematic of the magnesium unit cell showing two "opposite" $\{10-12\}$ twinning planes ( $\mathrm{C} 1$ and $\mathrm{C} 2$ in red) forming a twinning pair. Only the traces in the $\{0001\}$ plane for the other four twinning planes are shown for clarity; (b) The orientation of one parent matrix (black triangle) and corresponding six $\{10-12\}$ twinning variants (squares) in a $\{0001\}$ pole figure. Twin variants with the same colors (but different brightness) show twins from the same pair with a characteristic misorientation of $7.4^{\circ}$, while the two variants from any different pairs have also misorientations of either $60.0^{\circ}$ or $60.4^{\circ}$. 


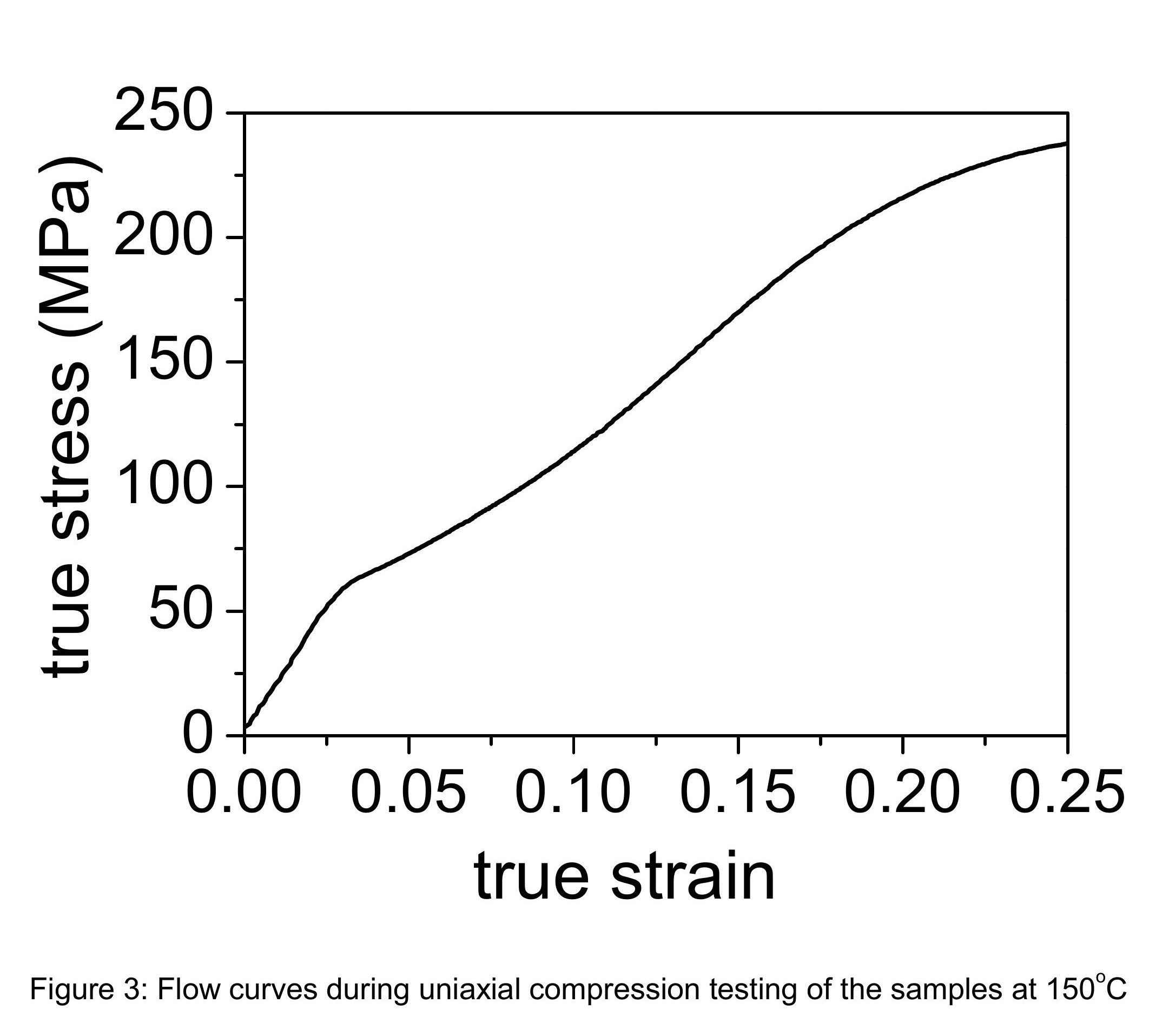

Figure 3: Flow curves during uniaxial compression testing of the samples at $150^{\circ} \mathrm{C}$

-

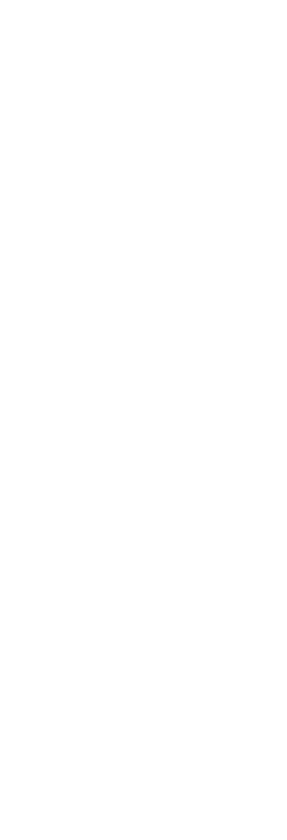



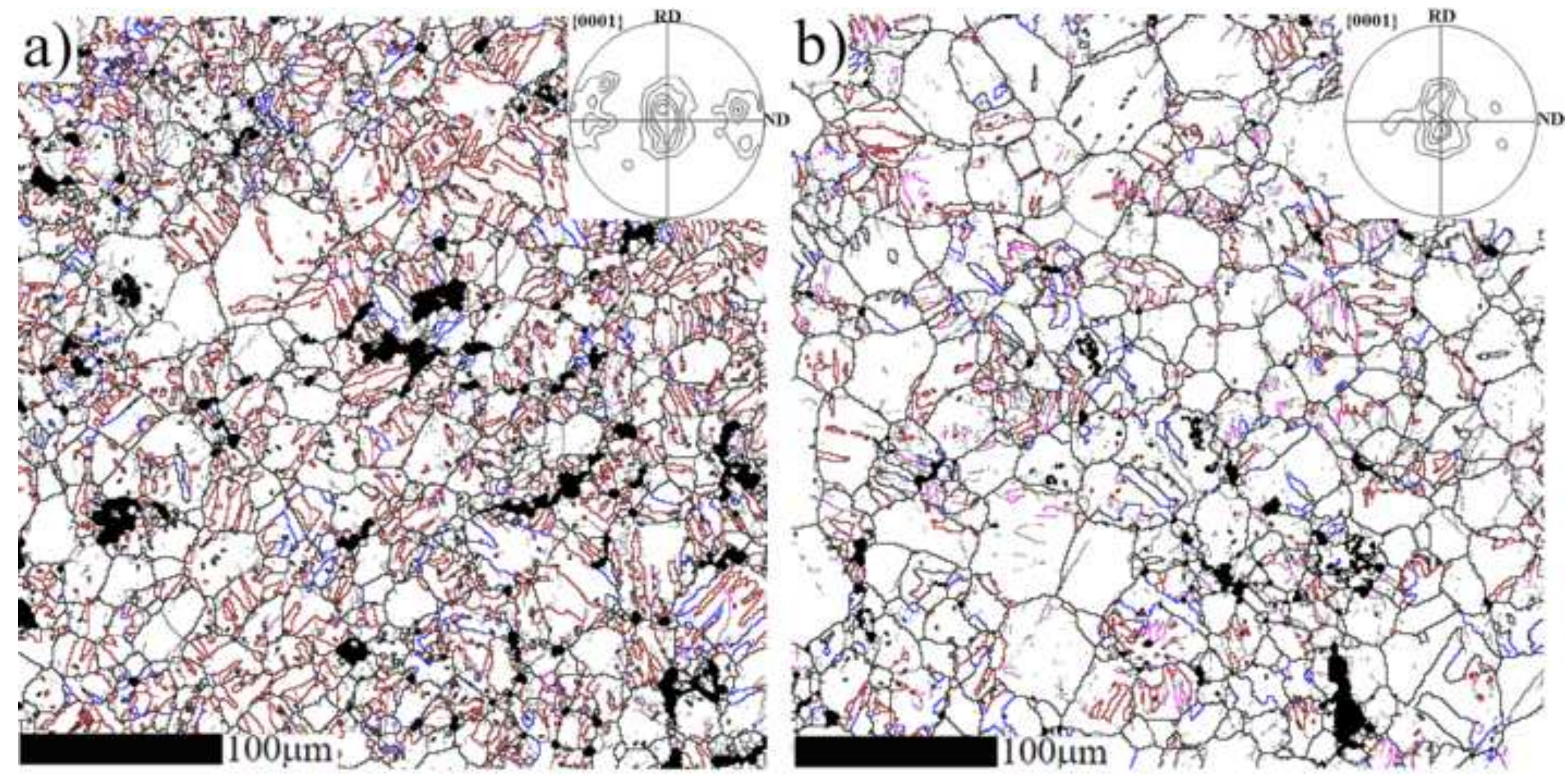

Figure 4: Microstructure and $\{0001\}$ pole figure (compression axis at the centre) for samples after uniaxial compression at $150^{\circ} \mathrm{C}$ to strains of: (a) $5 \% ;(b) 10 \%$. Red lines indicate $\{10-12\}$ twins with characteristic misorientation $86.4^{\circ}$; blue lines indicate boundaries between two twin variants from different pairs; purple lines indicate boundaries between two variants from the same twin pair; thin black lines indicate other boundaries with misorientations from $2^{\circ}$ to $10^{\circ}$; thick black lines indicate above misorientations $>10^{\circ}$. Solid black areas indicate non-indexed regions in the EBSD map. 

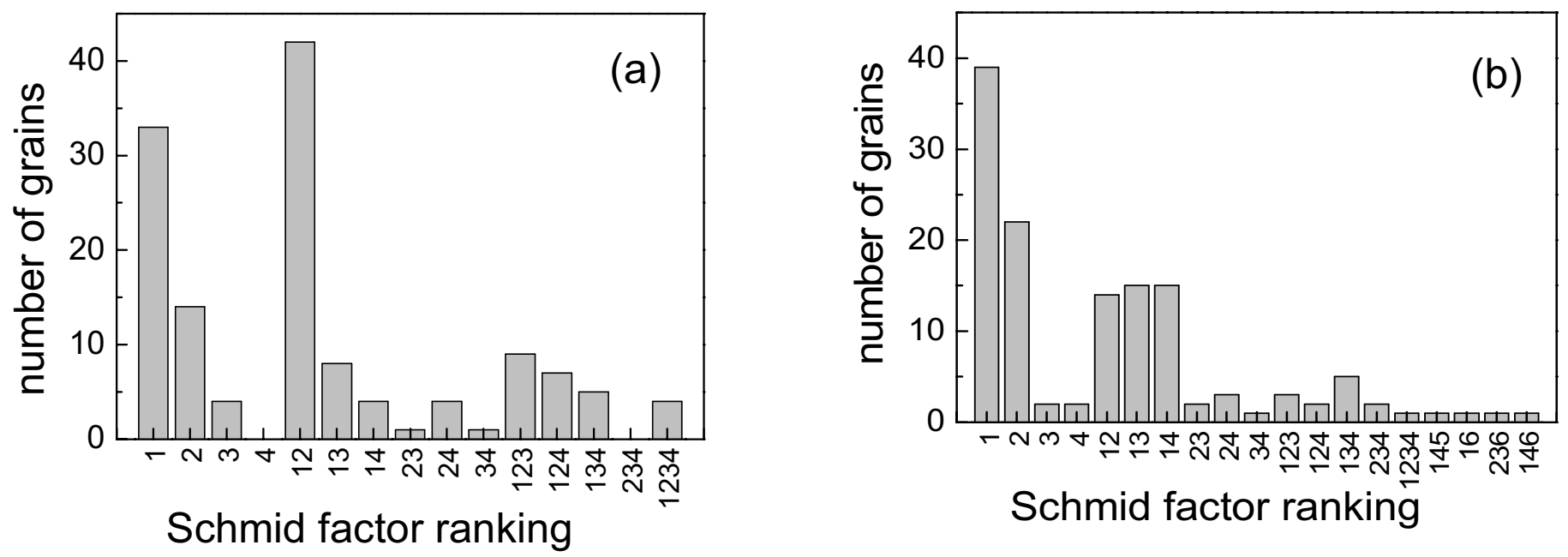

Figure 5: Ranking of Schmid factors for twin variants in samples deformed to strain of (a) $5 \%$, (b) $10 \%$. Example of labelling: Rank "1" represents a grain with a single twin variant with the highest SF; Rank "14" denotes a grain with two twin variants corresponding to the first and fourth highest Schmid factors 
(a)

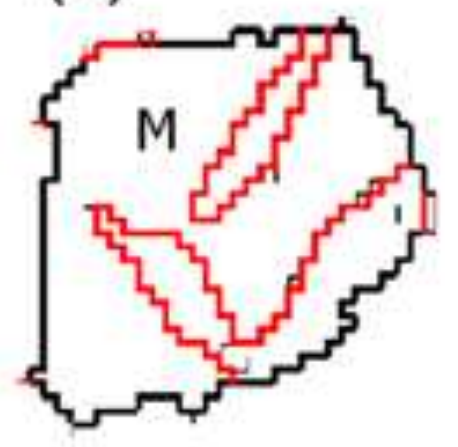

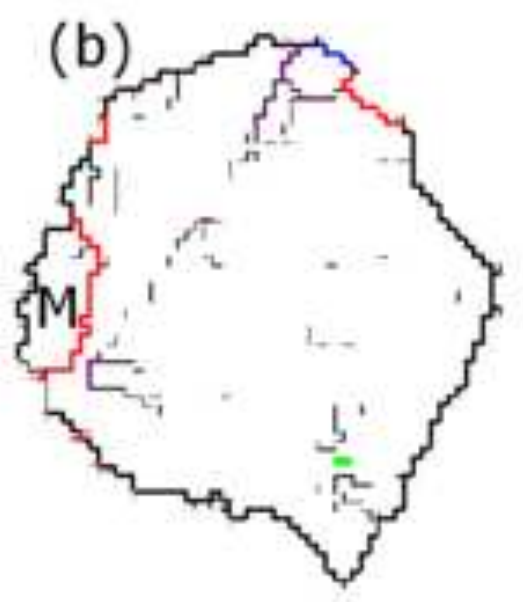

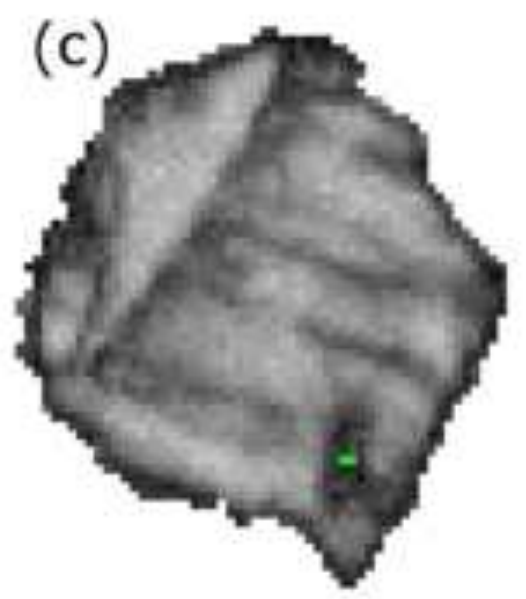

Figure 6: (a) Example EBSD boundary map of a grain at strain of $5 \%$ containing two twin variants from the same pair; (b) example EBSD boundary map of almost fully twinned grain at a strain of $10 \%$. In a few places $7.4^{\circ}$ $<1-210>$ boundaries are seen indicating abutment of two twins from the same pair. For both maps " $M$ " indicates the untwinned matrix; (c) EBSD band contrast map for same grain as (b) showing "ghost traces" corresponding to the two twin variants. Color code for misorientation same as Fig. 4. 

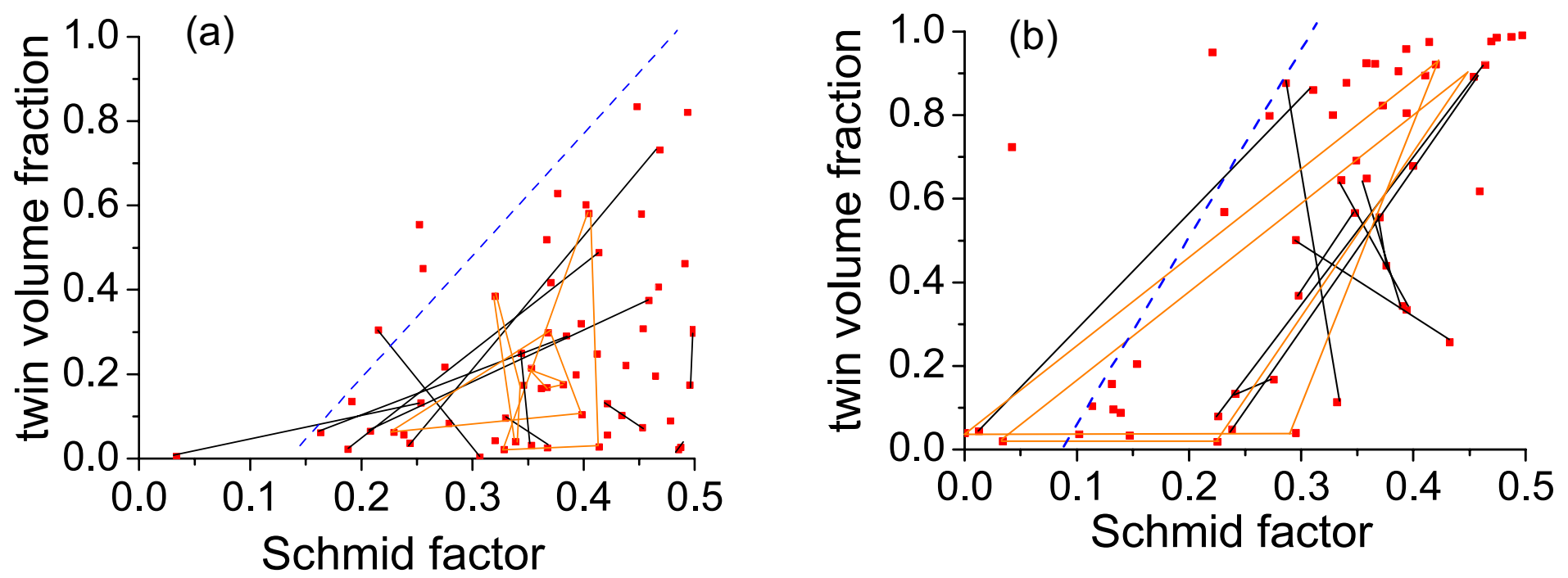

Figure 7: Relationship between twinning volume fraction and Schmid factor at strains of: (a) $5 \%$; (b) $10 \%$. Black lines connect the variants in grains containing two twinning variants; orange lines connect twins in grains containing three variants. An envelope giving the maximum twinning volume fraction as a function of Schimd factor is plotted in aqua. 

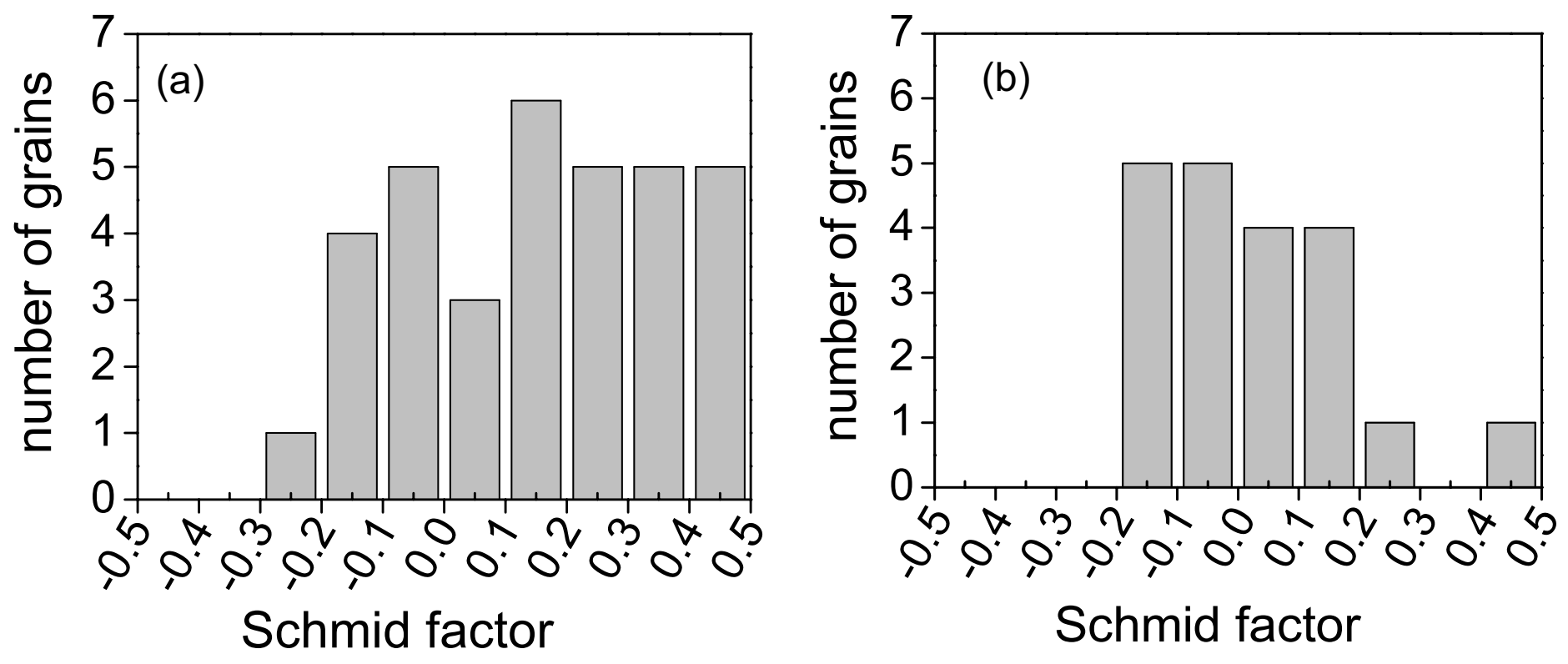

Figure 8: The maximum Schmid factor for untwined grains after compresion at $150^{\circ} \mathrm{C}$ to strains of: (a) $5 \%$; (b) $10 \%$. 


\section{a) compression axis}

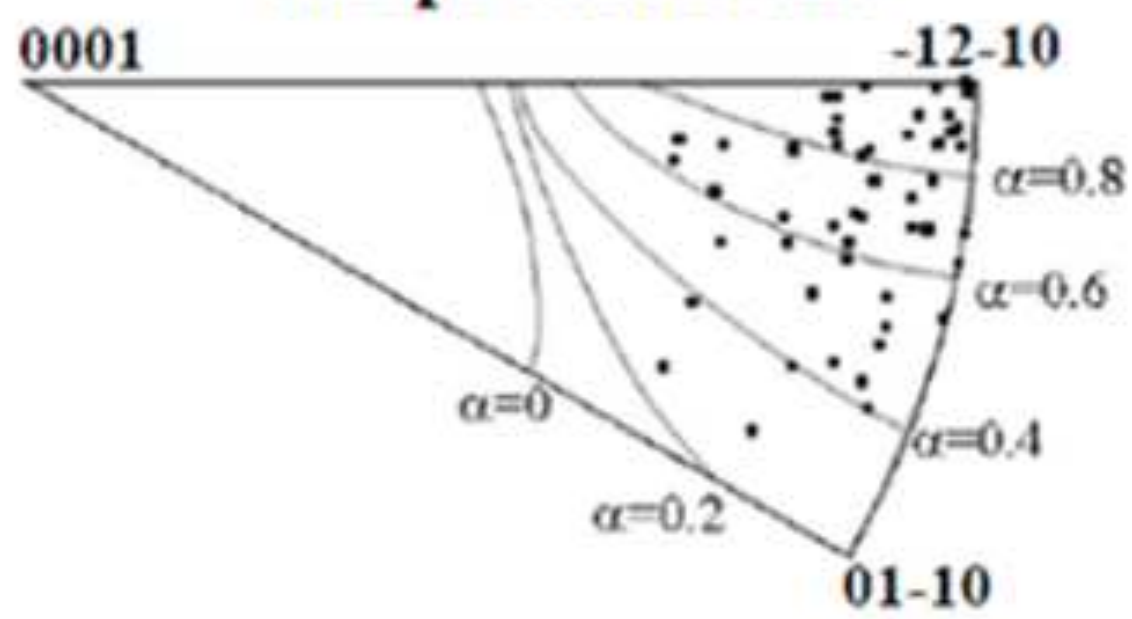

b) compression axis

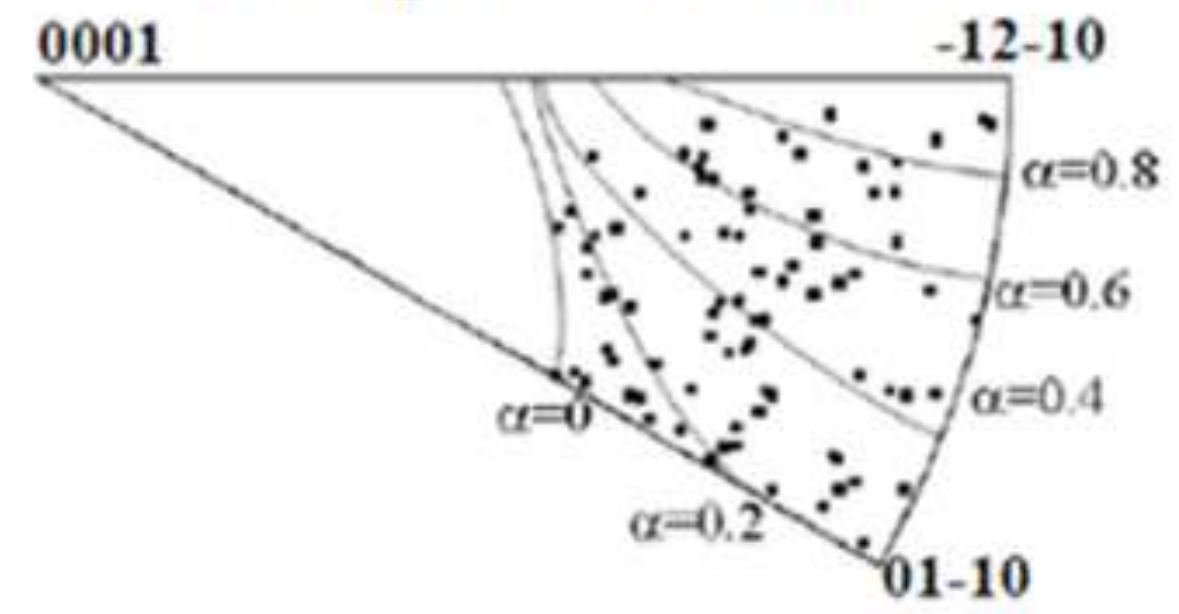

Figure 9: Distribution of grain orientations after 10\% deformation for :(a) grains containing twinning variants with third and/or fourth highest Schmid factor (SF); (b) grains only containing twinning variants with first and/or second highest SF. Contours of the ratio of fourth ranked SF to first ranked SF are also shown. 
a)

compression axis $-12-10$

b)

0001

compression axis $-12-10$
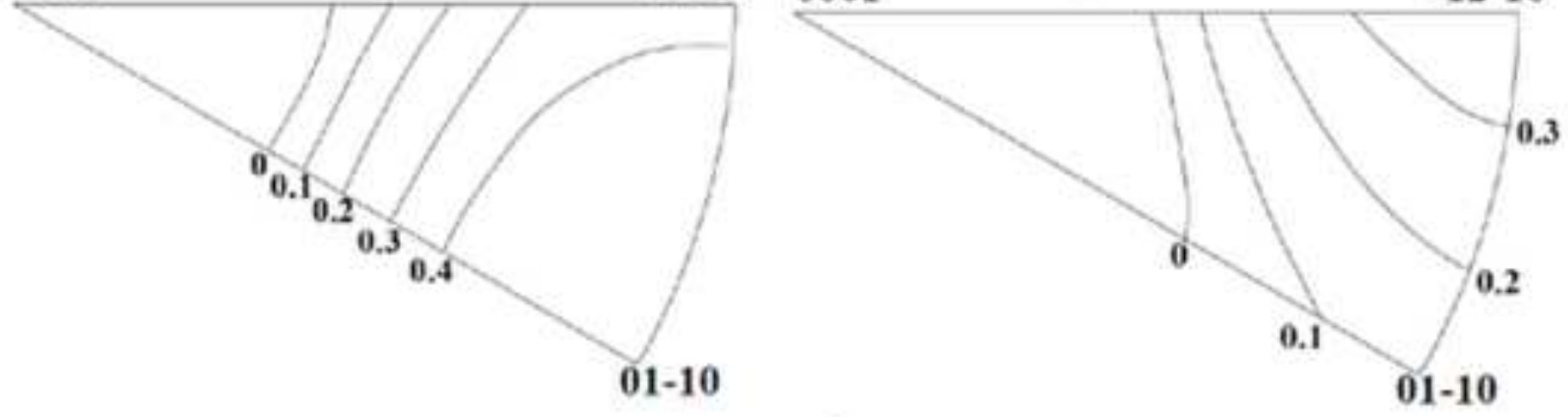

c)

compression axis

d)

$-12-10 \quad 0001$

compression axis
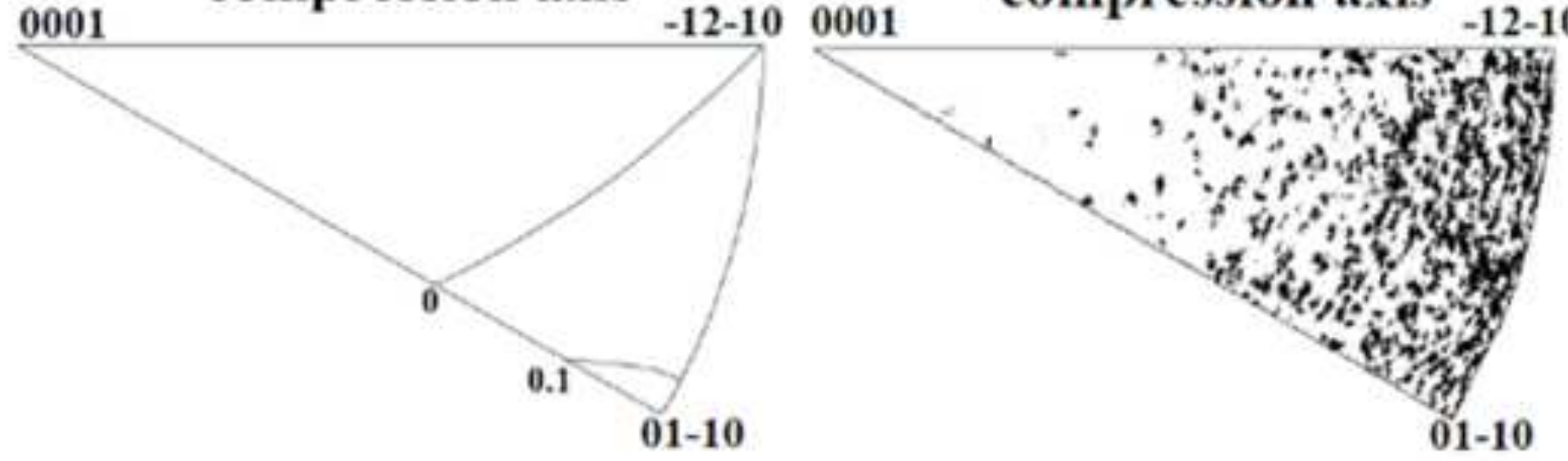

Figure 10: Inverse pole figures (compression axis direction) showing contour plots of the variation in Schmid factor (SF) value for the: (a) highest SF, (b) fourth highest SF, (c) sixth highest SF; (d) the orientations of grains for a sample of the initial material used in this study. 


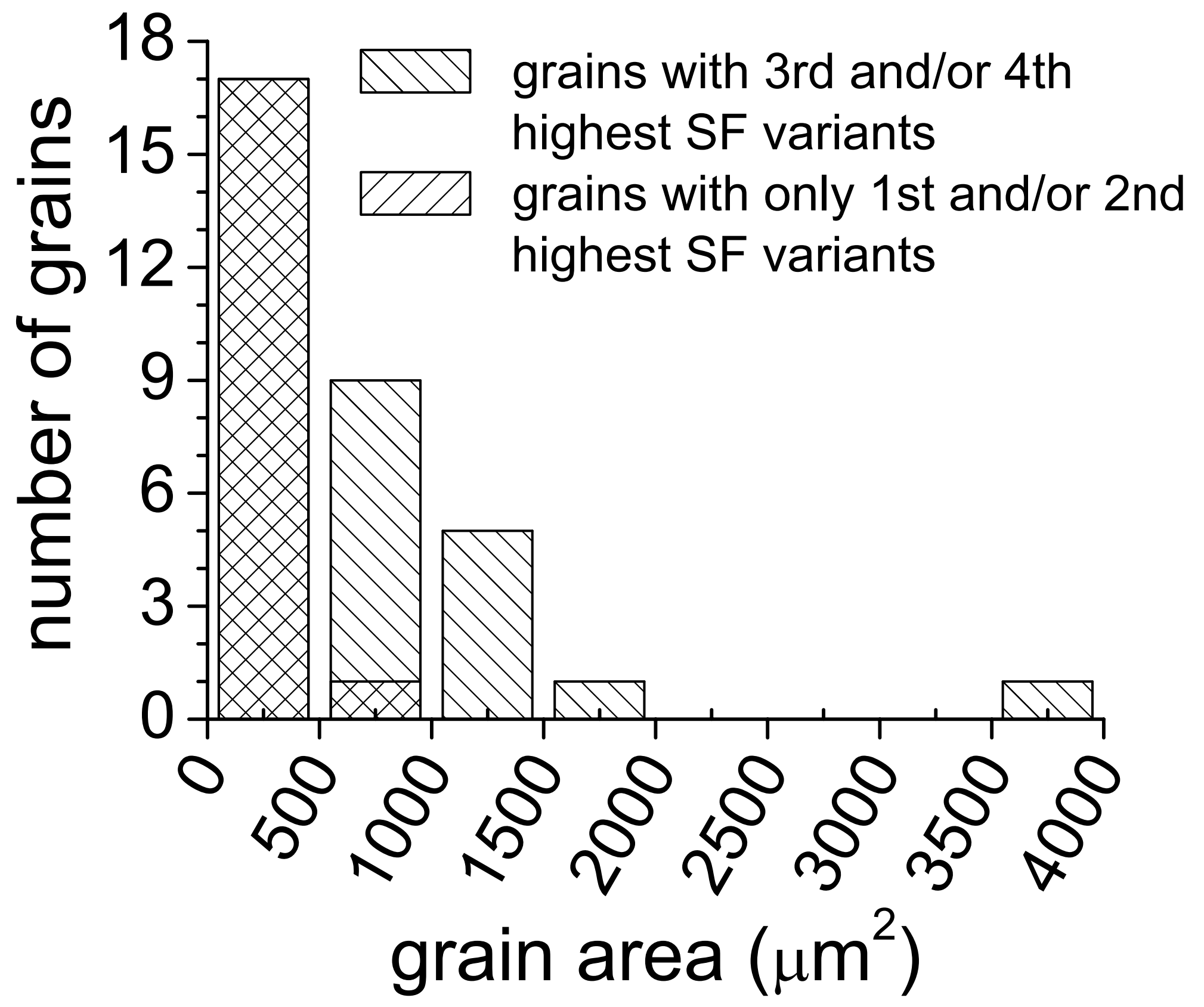

Figure 11: Distribution of grain area for all the grains with compression axis directions corresponding to an $\alpha$ value $>0.6$, where $\alpha$ is the ratio of the fourth ranked SF to the first ranked SF. 